it should not be more than from five to fifteen milliamperes. At this stage of the treatment the patient usually declares that he feels a decided local warmth at the negative pole. While maintaining the electrodes in their respective positions, the current is reversed some five or six times with the current reverser of the battery.

With the larger sponge electrodes general faradism is applied to every part of the body. One pole is placed at the nape of the neck or under the sacrum, while the other is passed successively over all the muscles causing in them a gentle but decided contraction. Many patients are over-sensitive, and in such it is advantageous for the physician to hold one pole of the battery in his left hand while he uses his right hand to administer the electricity with a gentle massage to the patient. This is more tiresome to the physician, and fortunately is necessary in only a small percentage of cases. I have used the method many times without feeling any bad effects from the electricity passing through myself. The advantages are that the physician can control the strength of the current better and a sensitive, nervous patient is not annoyed by any sudden shocks. While the spinal galvanism should not be prolonged beyond five minutes, as a rule, the general faradism is gradually increased in strength and time until the latter is an hour and the former produces almost painful contractions of the muscles.

In selected cases this simple method of administering electricity affords, after a few seances, a grateful feeling of rest, sleepinese and well being, which continues until in the end the whole constitution partakes of the improvement, and the patient looks and feels once more like a normal being. If after the firat two or three applications a restful feeling and a desire for sleep are not experienced, the physician's suspicions should be awakened. If a condition of over-excitability is produced he may be quite certain that his diagnosis is wrong, and the case is one not amenable to electric treatment.

With a proper knowledge of the qualities of the electric current, with more care in the making of the diagnoses and the selection of cases, and with an intel. ligent scientific application of the agent, electricity, in the treatment of the neuroses ought not to be abused as much as it is, but it ought to be used with a degree of success that will award it its due place among our therapeutic resources as one of the most powerful and effective in the conflict with disease.

4544 Lake Ave.

\section{A CASE OF LOSS OF BLOOD FROM THE RECTUM DUE TO MUCO-MEMBRANOUS ENTERO-COLITIS, AND TWO OF LEUKEMIA.}

Clinic delivered at the Jefferson Medical College Hospital. BY H. A. HARE, M.D.

Professor of Therapeutics and Materia Medica, Jefferson Medical College of Philadelphia.

PHILADELPHIA, PA.

The first patient is a man of 23 who is somewhat thin and anemic, and whose face shows the signs of mental depression and lack of nervous energy. His history is, that he was in perfect health until about two years ago, when he was suddenly seized with a severe hemorrhage from the bowels, the blood being quite bright in color. He tells us, however, that he has had no diarrhea at any time, but on the contrary suffers from obstinate constipation. There is nothing in his history which points clearly to the cause of these bloody movements, which have continued from time to time, generally once or twice a week ever since he was first taken ill. The frequent loss of blood has resulted in general debility and anemia. He tells us that an examination of his passages not only reveals blood but a large amount of mucus which appears in shreds or stringy masses. He often has much griping pain and flatulent distension, his appetite is variable and his diet is an irregular one because he is afraid to eat certain articles lest they produce an increase in his discomfort. Every few days he is seized with an attack of looseness of the bowels which seems as if it were an effort upon the part of the bowel to get rid of an excessive quantity of mucus and feces which have accumulated. His urine is normal.

When a case of hemorrhage from the bowels comes before you, the possibility of it arising from a number of different causes at once enters your mind. First, it is possible and most probable in such a case that the hemorrhages come from hemorrhoids, but the man tells us that he has no sign of any congestion of his hemorrhoidal veins. This does not, however, prove positively that the blood has not this origin, for the most severe cases of bleeding from the rectum are seen in those persons who have no large dilatations of the hemorrhoidal veins, but in their place have small arterial twigs in a dilated and hyperemic state and these little bunches of vessels containing arterial blood, when scraped or eroded by fecal matter give free vent to little arterial spurtings. A careless examination of the rectum might result in their being overlooked, but a careful examination with a speculum will often reveal their presence.

Relief may be obtained by touching them very lightly with the tip of a fine glass rod dipped in nitric acid, the resulting scar puckering the blood vessels and preventing further hemorrhage, or in other cases an application of nitrate of silver or of dilute nitric acid for its astringent effect does equally well. I have had this young man carefully examined, how. ever, and the report is that there is no sign of such bleeding points and we have therefore to look further for the cause of his trouble. Is it possible that the blood arises from a duodenal ulcer? I think not; for first, duodenal ulcer is a rather rare condition, and second, the blood which comes from such an ulcer is nearly always dark and tarry, through the action of the digestive juices of the bowel upon it, whereas the blood this man passes, he tells us, is quite bright and arterial in hue. Then, too, he has no pain in the neighborhood of the duodenum; such as is usually felt when ulcer of this portion of the alimentary canal is present and, finally, the blood is not well mixed with the fecal matter, but is well separated from it in the movements, although if it arose in the small intestine we would expect it to be thoroughly mixed with the stools. On the other hand, duodenal ulcer is far more frequent in males than in females. I think for the reasons given we may exclude the possibility of the blood arising from an ulcer in the duodenum.

The next cause to be thought of is cirrhosis of the liver. This condition, as is shown so well in the illustration of the results of cirrhosis of the liver in my book on "Diagnosis," results quite frequently in grave engorgement of the various abdominal viscera, 
sò that we frequently have hemorrhages from the stomach as the result of the enlargement of the gastric and esophageal veins and of the chronic catarrhal condition which results. Similarly it might be possible to have bloody movements from this cause as the result of the interference of the abdominal circulation and particularly from congestion of the hemorrhoidal plexus, but he has no history pointing to that most frequent cause of cirrhosis, alcoholism, nor does physical examination reveal any signs of this slow pathologic process in the liver. I think we may, therefore, exclude the possibility of the cause of his hemorrhages being hepatic cirrhosis. That he is not suffering from hemorrhages arising from old ulcers remaining after typhoid fever is proved by the fact that he has no history of this disease. $\mathrm{H}_{e}$ is, however, a markedly neurotic patient and the fact that he has these peculiar stringy movements, that he suffers from constipation with marked intestinal discomfort and with occasional attacks of diarrhea and from various spots of tenderness in the abdomen, which are not constant, leads me to conclude that he is suffering from ordinary muco-membranous entero-colitis. In some of these cases actual membrane seems to be passed from the bowel, but in the majority of them thick, tenacious strings of white mucus are passed with each movement. The proper treatment for this man is to institute, as closely as possible, what is known as the rest-cure treatment, which by improving the conditions of his nervous system, will indirectly cure this condition. His constipation must be overcome by the use of proper laxatives, such as cascara sagrada, to which may be added small quantities of lobelia to increase intestinal secretion, or if he has an attack of diarrhea this must be controlled by proper remedies. $\mathrm{He}_{\theta}$ must have a nourishing diet, which should be, however, free from potatoes, beans and similar articles which cause fermentation and the development of large quantities of wind in the bowel. Milk should be given him very cautiously until we are sure that he can digest it. In addition to this treatment his digestion should be aided by bitter tonics, by the use of pancreatin or pepsin, and, almost as important is the rest-cure. He should receive high injections of as hot water as he can bear, in each two liters of which there should be placed about 1.3 grams of the sulphocarbolate of zinc. Should no diarrhea be present we may place in the hot water from 2 to 4 grams of boric acid. His buttocks should be elevated before the injection is given, the fountain syringe should not be raised more than eighteen inches or two feet above the bowel, in order that the water will enter the bowel very slowly, and this treatment should be given at first every night and afterward at least three times a week. During the periods of his constipation gentle massage of the abdominal area may be carried out with great advantage, but it will have to be most gentle, particularly when it is first employed.

The other two cases are very much more important; they represent that not very common condition known as leukemia, or as it was formerly called, leucocythemia. In an earlier clinic I showed cases representing ordinary anemia, chlorosis and pernicious anemia, in all of which conditions the variations in the blood depended chiefly upon alterations in the character or number of the red blood corpuscles. These cases, however, represent but little change, comparatively speaking, in the red corpuscles, and very marked change in what are known as the white corpuscles or leucocytes.
The white blood corpuscles appeur in several forms: first, we have the small white blood corpuscle, scarcely as large as the ordinary red corpuscle, which is called a lymphocyte; this has a very large nucleus, which so completely fills the body of the corpuscle that there is no free protoplasm to be seen around its maryin. Next we have what is known as the large mononuclear leucocyte, a corpuscle considerably larger than the red corpuscle containing a large single nucleus which is surrounded by an area of transparent protoplasm. Next we have a white corpuscle known as a transitional leucocyte, in which the nucleus of the large mononuclear leucocyte looks as though it were undergoing some transitional change in its shape and character. This corpuscle is not, however, exceed. ingly important in diagnosis. Then we have what is known as the polymorphous or polynuclear leucocyte, a white blood cell which, as its name indicates, con. tains either several nuclei or a nucleus of very irregular shape (polymorphous). This corpuscle contains in its protoplasm a number of fine granules which take certain neutral stains and are therefore known as neutrophiles. In addition we find in normal blood another white blood corpuscle, generally polynuclear in character, in the protoplasm of which may be found large granules, much larger than those just described, which readily take an acid stain such as eosin. They are called eosinophiles. At one time the presence of those cells was considered of importance in the diagnosis of leukemia, but they are now considered not so important. These, then, are the white corpuscles which are found in normal blood. What are the changes which we find in leukemia? Before I describe them I must recall to your mind that we have two forms of leukemia; first, spleno-medullary leukemia, which is by far the most frequent type. In this disease we have great enlargement of the spleen and liver and marked pathologic changes in the medulla of the bones and, as a result of these changes, it is supposed that we have developed an excessive number of large mononuclear leucocytes, and at the same time another white corpuscle not found in normal blood is seen which appears as a myelocyte, a corpuscle of very large size with a very large staining nucleus, usually situated toward one side of the cell and in the protoplasm of this corpuscle are to be found numerous fine granules. The presence of these two corpuscles and the mononuclear cells in excess and the development of the myelocytes affords us strong evidence of the presence of spleno-medullary leukemia, particularly if we find in association with these signs the dyspnea of anemia, the pallor, retinal hemorrhages, possibly hematuria and the marked enlargement of the spleen which are so characteristic. The older of these two patients presents just these signs and symptoms. An additional sign of leukemia may be, perhaps, considered in the marked decrease in polynuclear leucocytes which are present, and this enables us to separate leukemia from other cases in which we simply have excessive leucocytosis, because the polynuclear leucocytes are those which are increased in excessive leuoocytosis but decreased in leukemia. Repeated examinations of this man's blood show the following changes: September 30 he had 2,458,000 red corpuscles, 28 per cent. of hemoglobin and 368,750 white cells, the proportion of white to red being 1 to 6. Two months later under ascending doses of arsenic his hemoglobin had risen to 50 per cent., his red corpuscles, however, numbered only $2,048,000$, but 
the white corpuscles had been decreased to 93,750 , or 1 to 23.

Today his hemoglobin equals 63 per cent., his red corpuscles $4,000,000$ and his leucocytes only 19,000 . The proportion therefore is 1 to 210 , which approximates the normal proportion very much more closely than the earlier count. The blood also shows that the myelocytes are present in the proportion of from 8 to 13 per cent., and it is a noteworthy fact that his polynuclear leucocytes, which are usually decreased in this disease, amount to from 51 to 66 per cent., which is about the number usually found in normal blood. His spleen is found to be greatly enlarged. It extends almost to the anterior superior spine of the ilium and in the middle line as far as the umbilicus, but his color has greatly improved and there are many men who would appear far more anemio than he, although they would consider themselves as being in perfect health.

The second case is far more interesting because much more rare, representing as it does a case of possible lymphatic leukemia. It is that of a young man of 23 , a barber by occupation, who is intensely pale and yellow looking. An examination of his chest and arteries shows loud anemic murmurs, his spleen is enlarged to even greater extent than the patient first brought before you and we have records of his blood over many months. October, 1895, an exam. ination of his blood showed that his hemoglobin was 30 per cent., his red blood corpuscles 2,060,000 and his leucocytes 8000 . He has been in hospital off and on since that time and we have a great many counts of his blood. His case is not a typic one, but I think we can say that it is one of lymphatic leukemia. At the present time his hemoglobin is 33 per cent., his red corpuscles the same as before and the leucocytes 6200. While the red corpuscles are somewhat decreased, as they frequently are in leukemia, the white blood corpuscles are not increased as is usual; indeed, this proportion between white and red is often seen in health. It is only when we consider his various corpuscles that we have much light thrown upon the diagnosis. We then find that his lymphocytes, which are ordinarily present in comparatively small numbers, amount to 84 per cent., that polynuclear leucocytes, which usually make up a large proportion of the white cells, onty amount in this patient to 8 per cent., while the mononuclear leucocytes amount to 4 per cent. There is also absent in his case one other frequent sign of lymphatic leukemia, namely, very great enlargement of the lymphatic glands all over the body. This boy has no such enlargement, but his lymphocy tosis, combined with his profound anemia, the fact that he has had hematuria, that he has hemorrhages in his retina and that he is intensely anemic and pallid without a corresponding loss of flesh, seem to make the diagnosis fairly clear.

In many of these cases of leukemia we find in addition to a decrease in the number of red corpuscles, an abnormal red blood corpuscle which possesses a nucleus, this corpuscle being called a normoblast and sometimes also possessing ameboid movement. In the presence of a patient suffering from multiple enlargement of the lymphatic glands, a symptom which this boy might present, it would be incorrect for you to jump to the conclusion that he was suffering from lymphatic leukemia, because a similar enlargement is seen in what is known as Hodgkin's disease or pseudo-leukemia and multiple tuberoular infection. nourishing diet, the meeting of ourrent symptoms which have seemed of most importance, and ascending doses of arsenic until they reached the physiologic limit of the drug.

\section{FIVE CASES OF CHOLELITHIASIS.}

BY BAYARD HOLMES, M.D.,

PROFESSOR OF SURGERY IN THE COLLEGE OF PHYSICIANS AND SURAEONS OF CHICAGO, THE MRDICAL SCHOOL OF THE UNIVERSITY OF ILLINOIS.

The following cases are reported because each of them contains at least one point of real surgical interest:

Case 1.-Indistinct pain in the abdomen, with sick headache; the morphin habit; empyema of the gall bladder; cholecystostomy; recovery. The patient was a physician, 45 years of age, who came to Dr. Waugh's sanitorium for the treatment of the morphin habit. He was of very regular habits, used no tobacco or alcohol and had no significant previous history. $\mathrm{He}$ had indistinct pain in his back and abdomen for several years and was accustomed to take small doses of morphin to relieve this distress. These doses increased, however, in frequency and size until he found himself, under one pretext or another, taking larger and larger doses, up to 0.45 or 0.52 grams a day. He was bright and had been able to follow his pratctice, which was large and diversified. On coming to the sanitorium he stood the treatment well for a week or two, when without apparent cause and without any very great increase in this indistinct pain in his back he gradually began to have an evening rise of temperature, with some sweat. At length symptoms of peritonitis, distension of the abdomen and tympanites appeared, and it was discovered that his right abdominal region was filled by a tumor six inches long and three or four inches wide. It was so large a tumor that it was at first looked upon as a peri-appendicular abscess. In the course of twenty-four hours the eymptoms were so grave that I was called in to open the abscess. This was Dec. 16, 1893. The patient at this time was only partially conscious; his abdomen was slightly tympanitic; the bowels had been freely moved by an enema ; the patient's temperature was 102 , his pulse 120 . He had never had any paroxysmal pain, but complained of a deep pain in the back, which was relieved by the use of morphin. In examining the eyes the pupils did not show the effect of the morphin ; the tongue was coated typhoid-like; the lips dry and parched; the skin hot and dry; the respirations rapid and superficial, as in peritonitis; the heart's action was perfect, but rapid; the abdomen was slightly distended, and in the right side was found a tumor, round, smooth and pressing up against the abdominal wall, which tumor I readily recognized as a greatly distended gall-bladder or possibly a pyonephritic tumor. His urine was examined and found to contain a trace of albumin and was otherwise highly concentrated. He urinated voluntarily. The case was considered one of great gravity, and an immediate operation was undertaken. The abdomen was opened by an incision along the outer border of the right rectus muscle two and ope-half inches long. The tumor showed itself in the wound unattached to the viscera. The first effort, however, to examine it resulted in rupture of the sac and the discharge of a large quantity of thick pus. The walls of the tumor were instantly grasped in several pair of forceps and the tumor pulled out into the wound. By means of several silk sutures, it was attached to the margins of the wound and the edges were trimmed off, making a large opening in to the gallbladder. The finger was passed down to the cystic duct, but on account of the necrotic condition of the sac it was considered unadvisable to make any attempt to sound the cystic duct.

During the following twenty-four hours the temperature fell to 99 and rose again at the end of the second day to 101, after which it gradually declined until the twenty-fifth day. Upon the eighth day the stone appeared at the mouth of the wound and was with some difficulty removed. It was half an inch in diameter and about an inch long, being pear-shaped and having a facet at one end, showing that another stone had been in contact with it. This was dislodged a few days later. The patient made an uninterrupted recovery, with one exception. On the tenth day after the operation the usual irrigation of the wall of the gall-bladder was undertaken by the nurse, but the mouth of the fistulous opening into the gall-bladder had now become so small that the irrigator point did not allow a free return of the water from the cyst, and as a result the water probably flowed through the cystic into the hepatic duct and carried back with it the pus from the gall-bladder. The patient com-
plained of a sudden dreadful pain, quickly went into a collapse 